\title{
Wearable device for automatic detection and monitoring of freezing in Parkinson's disease
}

\author{
Michelle Millán ${ }^{1 *}$, Hiram Cantú ${ }^{2}$ \\ ${ }^{1}$ Departamento de Ingeniería Biomédica, Vicerrectoría de Ciencias de la Salud, Universidad de \\ Monterrey, 66238 San Pedro Garza García, Nuevo León, México \\ ${ }^{2}$ Departamento de Ingeniería Biomédica, Vicerrectoría de Ciencias de la Salud, Universidad de \\ Monterrey, 66238 San Pedro Garza García, Nuevo León, México
}

\begin{abstract}
Freezing of gait (FOG) in Parkinson's disease (PD) is described as a short-term episode of absence or considerable decrease of movement despite the intention of moving forward. FOG is related to risk of falls and low quality of life for individuals with PD. FOG has been studied and analyzed through different techniques, including inertial movement units (IMUs) and motion capture systems (MOCAP), both along with robust algorithms. Still, there is not a standardized methodology to identify nor quantify freezing episodes (FEs). In a previous work from our group, a new methodology was developed to differentiate FEs from normal movement using position data obtained from a motion capture system. The purpose of this study is to determine if this methodology is equally effective identifying FEs when using IMUs. Twenty subjects with PD will perform two different gait-related tasks. Trials will be tracked by IMUs and filmed by a video camera; data from IMUs will be compared to the time occurrence of FEs obtained from the videos. We expect this methodology will successfully detect FEs with IMUs' data. Results would allow the development of a wearable device able to detect and monitor FOG. It is expected that the use of this type of devices would allow clinicians to better understand FOG and improve patients' care.
\end{abstract}

\section{Introduction}

Parkinson's disease (PD) is defined as a neurological condition that evolves progressively and it is characterized by the appearance of hypokinesia, akinesia, tremor, rigidity and postural instability. These movement disturbances are associated with gait altered patterns, such as short and slow steps. In most of the cases, PD tend to affect elderly, which results in performance complications during specialized motor tasks like walking, writing and talking [1].

In addition to these symptoms, some individuals with PD experience episodic movement arrests that usually occurs in advanced stages of the disease, better known as freezing. Freezing is more commonly studied in gait (freezing of gait, FOG) [2-9] and it is described

\footnotetext{
* Corresponding author: michelle.millan@udem.edu
} 
as a short-term episode of absence or considerable decrease of movement despite the intention of moving forward [8]. However, $80 \%$ of the PD patients will never go through FOG at any stage of the disease [10].

This phenomenon is related to risk of falls and low quality of life for individuals with PD. While the disease progresses, FOG becomes more frequent and disabling usually leading to falls [10-13]. One of the most feared consequences from falls are hip fractures which typically lead to nursing home admission. Hip fractures are related to a high mortality and high morbidity in PD [14]. According to Hosseinzadeh and colleagues' study [15], PD almost tripled the risk of hip fracture, implying a direct association between PD and fracture risk.

Currently, there is only one tool validated to identify FOG, a self-assessment questionnaire in which PD patients self-report how often they experience freezing $[16,17]$. This tool is highly sensitive in identifying those individuals with PD who experience freezing (freezers) from those who do not (non-freezers). However, the Freezing of Gait Questionnaire (FOGQ) is subjective and does not help to quantify the specific time when this phenomenon occurs.

Based on the FOG definition previously described, it is suggested that the influence of freezing can vary from temporary gait pattern disruptions to a complete gait cycle arrest [18]. Therefore, several objective approaches that fit the definition have been used to identify and study freezing from different perspectives (i.e. kinematics, kinetics) to minimize its effects [3], [11], [19-21]. From all these approaches, complete movement arrests and initial amplitude decreases are the most common criteria used to identify freezing in PD [22-26], two different methodologies that raise the need of a standardized method to ensure the same phenomenon is being studied and allow comparisons across studies.

The use of inertial movement units (IMUs), such as accelerometers, has been a popular method to identify and quantify FOG in real time along with robust algorithms. Tripoliti and colleagues [27] identified FOG by analyzing data obtained from accelerometers during gait tasks using a Random Forests classification algorithm with $81.94 \%$ sensitivity. Moreover, Cole and colleagues [28] created a Dynamic Neural Network for freezing detection using accelerometers during unscripted activity reaching $83 \%$ of sensitivity. Rezvanian and Lockhart [29] used the continuous wavelet transform (CWT) to decompose acceleration data obtained from IMUs by taking into account the frequency and time domains, reaching a sensitivity of $84.9 \%$.

Although many efforts have been made in order to study FEs, there is no standardized methodology to identify nor quantify them, which prevent comparison among different studies. Despite the fact that many studies coincide between technologies, IMU methods are often unclear. If IMUs are expected to be used as a substitute for motion capture systems (MOCAP), IMUs methods are required to be reliable and reproducible [30].

Based on our recent results using position data obtained from MOCAP [31], it was shown that a freezing method based on complete movement arrests rather than amplitude decrease better discriminates freezing from normal movement during a gait related task. Moreover, higher sensitivity $(88.3 \%$ ) was obtained by using this new method that considers: (i) complete movement arrests as freezing criteria, (ii) 0.5 seconds as the minimum duration of a freezing episode, (iii) a minimum time of 2 seconds to overcome a freezing episode, and (iv) a robust algorithm that considers both, time and frequency characteristics of the signal (CWT). 
The purpose of this study is to determine whether this method is equally effective detecting FEs when using IMUs (i.e. acceleration data). Moreover, we aim to define the most optimal anatomical landmark to place IMUs that would better differentiate freezing from normal movement. We define sensitivity as the probability for our method to identify FEs when FEs occur, and specificity as the probability for our method to identify normal movement when FEs do not occur. To address these objectives, individuals with PD will perform an experimental protocol, which includes specific tasks, in order to obtain data from IMUs. Twenty participants with PD will be recruited: 10 freezers and 10 non-freezers, classified according to their FOGQ results. We assumed that the proposed tasks will trigger FEs in individuals with PD.

We expect that (i) the methodology used in data obtained from motion capture system will be equally effective to identify and quantify FEs in data obtained from IMUs, also with the obtained results it will be possible to (ii) develop a methodology to predict FOG using IMUs, (iii) develop a wearable device able to detect and monitor FOG by the implementation of the proposed methodology, and (iv) objectively evaluate FOG quantification.

Movement analysis of PD individuals with FOG focuses, among other purposes, on identifying and quantifying FOG through different methodologies. Development and implementation of new methodologies for FOG detection enhances the design and creation of wearable devices capable of detect and monitor FOG. As a result of this research, a new methodology could be implemented in a wearable device for detection and monitoring of FOG in PD patients. The study and analysis of FOG is essential for the development of technologies, which can provide novel insight to the experts, such as neurologists, geriatricians, rehabilitation specialists, physiotherapists and nurses, who work closely with FOG.

\section{Methodology}

\subsection{Subjects}

Twenty participants with PD (10 reporting FOG, and 10 without FOG) will be recruited for this study. Participants will be classified as freezers if a score $\geq 1$ is met on item 3 of the Freezing of Gait Questionnaire (FOGQ): “do you feel that your feet get glued to the floor while walking, making a turn or when trying to initiate walking?" [16]. Freezers and nonfreezers will be matched for disease severity. The inclusion criteria will be: (i) idiopathic PD, (ii) age between 50 and 80 years, (iii) Hoehn and Yahr stage I, II, III, or IV, where bilateral impairment movements can be identified, but individuals are still able to walk or stand unassisted [31], and (iv) use of levodopa medication. The exclusion criteria will be: (i) mild cognitive impairment defined by a score $\leq 25 / 30$ on The Montreal Cognitive Assessment (MoCA), (ii) neurological, orthopedic, or muscular disorders other than PD, (iii) currently having implanted deep brain stimulation (DBS) electrodes or (iv) history of diabetes.

A control group of 10 healthy age- and sex-matched participants will also be recruited. Control subjects (i) presenting history or evidence of neurological, orthopedic, or muscular disability, (ii) self-reporting postural impairments or unexplained falls in the last year, (iii) taking medication that might affect balance (i.e. ototoxic drugs such as antidepressants, antiseizure, diuretics), (iv) complaining of paresthesia characterized by the sensation of tingling skin and/or a limb falling asleep, or (v) with a history of diabetes will be excluded from this 
study. All participants will provide written informed consent prior to their participation in the study.

\subsection{Clinical evaluation}

First, participants with PD will be clinically assessed with (i) a medical history questionnaire, (ii) the MoCA test, (iii) the Unified Parkinson Disease Rating Scale Part 3 (UPDRS-III), (iv) the Hoehn and Yahr (H\&Y) scale, and (v) the FOGQ.

\subsection{Experimental protocol}

The experimental protocol includes two different tasks. First task consists of performing a stepping-in-place (SIP) task, a gait-like activity that consists on participants raising the legs alternately and continuously at a comfortable height (see Fig. 1). The SIP has been successfully used to identify freezing episodes in individuals with PD [32]. Participants will be asked to perform the task at a comfortable and self-selected pace that will be obtained from practice trials.
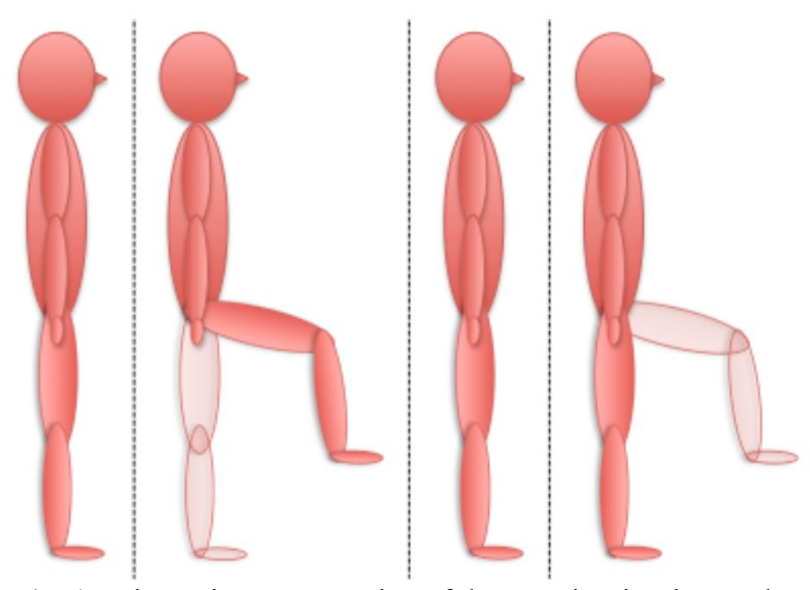

Fig. 1. Schematic representation of the stepping-in-place task.

The experimental protocol includes two trials of the SIP task, the first of 30 seconds and the second of 120 seconds duration. Before starting the task, participants will be provided with a 10-beat auditory feedback from a metronome set at the self-selected pace and they will be asked to memorize and perform the task following this rhythm.

Second task consists of performing different activities of daily life. As shown in Fig. 2, participants will (a) stand up from a chair, (b) walk straight for $6 \mathrm{~m}$ in a designated path of $50 \mathrm{~cm}$ wide, (c) turn $180^{\circ}$, (d) return to the chair while walking the $6 \mathrm{~m}$ and, (e) sit for 1 minute in the chair. According to Schaafsma and coleagues [33] this type of activities can trigger FOG. 
(b)

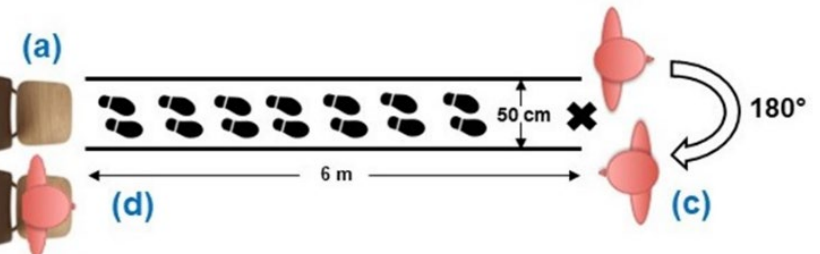

(e)

Fig. 2. Schematic representation of the daily life activities task.

Video data will be obtained by filming each session of the experimental protocol with a video camera placed in the frontal and sagittal plane. Kinematic data will be recorded during the whole trials and 5 seconds before initiating the SIP task in order to have a quiet stance baseline.

\subsection{Data acquisition}

Spatiotemporal measures of gait (accelerations) will be obtained from Trigno Avanti Sensors (Trigno $^{\text {TM }}$ Wireless Biofeedback System, Delsys Inc., Natick, MA). Participants will wear 8 wireless IMUs (triaxial accelerometers, gyroscopes and magnetometers) on the back and the lower limbs (thoracic and lumbar region, bilateral upper thigh, bilateral lower shank, and bilateral feet) as shown in Fig. 3.

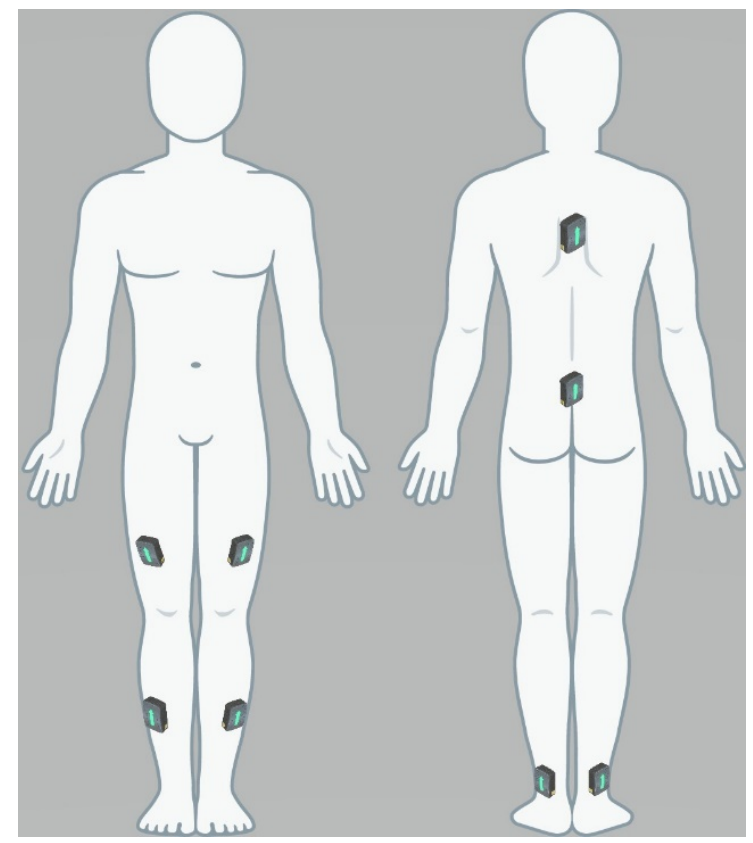

Fig. 3. Sensors placement. 


\subsection{Data analysis}

A freezing episode will be defined as a period lasting at least 0.5 seconds of movement arrest, in which one or both limbs display no movement. Moreover, a minimum time of 2 seconds between two freezing episodes will be required to consider it as 'normal movement'.

A pair of trained observers will inspect visually and independently inspected the videos for each participant in all trials to freezing occurrence. They will identify the number of FEs, duration time and the time when they occur. If no agreement is met between observers, videos will be reviewed by each of them.

Automated detection of FEs will be performed in 3D acceleration data obtained from each of the IMUs with the method previously described by computing the algorithm in Matlab (MathWorks, Massachusetts, USA). Number, duration time, and time of occurrence of the FEs will be obtained. Differences between the acceleration data and the video data (reference) then will be calculated for each outcome measurement.

\subsection{Statistical analysis}

For all the statistical analysis, significance levels of .05 will be used. Inter-rater reliability of the values reported by each observer prior to reaching consensus will be assessed using Cohen's kappa for the rating of the number, duration and time of occurrence of the freezing episodes. The differences of number and total duration of freezing episodes between the videos and acceleration data will be compared by means of a non-parametric repeated measures Friedman test for equal sample sizes. The accuracy to identify time of occurrence of a freezing episode between the videos and the acceleration data will be compared using a non-parametric repeated measures Cochran's Q test for unequal sample sizes. If significant differences are found in non-parametric tests, Wilcoxon post-hoc comparisons will be performed to determine pair-wise differences with Bonferroni corrections. The accuracy to identify freezing episodes on the acceleration data and on every different anatomical landmark will be described by the area under the receiver operating characteristic (ROC) curve in terms of sensitivity and specificity. Sensitivity refers to the probability that the algorithm applied to the acceleration data detects a freezing episode when freezing is present, while specificity indicates the probability that the algorithm detects normal movement when there is no freezing.

\section{Conclusion}

Currently, this research is underway. The proposed methodology will be applied in data obtained from IMUs in order to identify and quantify FOG in individuals with PD. Further analyses from IMUs data, as well as data from video recordings, will be used to determine if the proposed methodology is effective identifying FOG. From the obtained information, we expect to determine the optimal anatomical placement of the IMUs that better discriminate FOG from normal movement. It is expected that the closer the IMU is to the ground (e.g. ankle and/or heel) the better would be in identifying FOG. Results would allow the design of a wearable device prototype, which integrates the proposed methodology. These devices would allow the monitoring of individuals with PD who suffer FOG in order to reduce risk of falls, and also provide novel knowledge to help clinicians to tackle with improved rehabilitation therapies and medicine doses. 


\section{References}

1. M. E. Morris, F. Huxham, J. McGinley, K. Dodd, and R. Iansek, "The biomechanics and motor control of gait in Parkinson disease," Clin. Biomech., vol. 16, no. 6, pp. 459-470, (2001)

2. Q. J. Almeida and C. A. Lebold, "Freezing of gait in Parkinson's disease: A perceptual cause for a motor impairment?," J. Neurol. Neurosurg. Psychiatry, vol. 81, no. 5, pp. 513-518, (2010)

3. K. A. Ehgoetz Martens, F. Pieruccini-Faria, and Q. J. Almeida, "Could Sensory Mechanisms Be a Core Factor That Underlies Freezing of Gait in Parkinson's Disease?," PLoS One, vol. 8, no. 5, (2013)

4. U. M. Fietzek, J. Zwosta, F. E. Schroeteler, K. Ziegler, and A. O. Ceballos-Baumann, "Levodopa changes the severity of freezing in Parkinson's disease," Park. Relat. Disord., vol. 19, no. 10, pp. 894-896, (2013)

5. Y. Kwon et al., "A practical method for the detection of freezing of gait in patients with Parkinson's disease," Clin. Interv. Aging, vol. 9, pp. 1709-1719, (2014)

6. S. H. G. Mensink et al., "Additional weight load increases freezing of gait episodes in Parkinson's disease; an experimental study," J. Neurol., vol. 261, no. 5, pp. 999-1008, (2014)

7. A. Nieuwboer and N. Giladi, "Characterizing freezing of gait in Parkinson's disease: Models of an episodic phenomenon," Mov. Disord., vol. 28, no. 11, pp. 1509-1519, (2013)

8. J. G. Nutt, B. R. Bloem, N. Giladi, M. Hallett, F. B. Horak, and A. Nieuwboer, "Freezing of gait: Moving forward on a mysterious clinical phenomenon," Lancet Neurol., vol. 10, no. 8, pp. 734-744, (2011)

9. J. Spildooren, S. Vercruysse, K. Desloovere, W. Vandenberghe, E. Kerckhofs, and A. Nieuwboer, "Freezing of gait in Parkinson's disease: The impact of dual-tasking and turning," Mov. Disord., vol. 25, no. 15, pp. 2563-2570, (2010)

10. B. R. Bloem, J. M. Hausdorff, J. E. Visser, and N. Giladi, "Falls and freezing of Gait in Parkinson's disease: A review of two interconnected, episodic phenomena," Mov. Disord., vol. 19, no. 8, pp. 871-884, (2004)

11. J. V. Jacobs, J. G. Nutt, P. Carlson-Kuhta, R. Allen, and F. B. Horak, "Dual tasking during postural stepping responses increases falls but not freezing in people with Parkinson's disease," Park. Relat. Disord., vol. 20, no. 7, pp. 779-781, (2014)

12. G. K. Kerr, C. J. Worringham, M. H. Cole, P. F. Lacherez, J. M. Wood, and P. A. Silburn, "Predictors of future falls in Parkinson disease," Neurology, vol. 75, no. 2, pp. 116-124, (2010)

13. S. Vercruysse et al., "Explaining freezing of gait in Parkinson's disease: Motor and cognitive determinants," Mov. Disord., vol. 27, no. 13, pp. 1644-1651, (2012)

14. L. Coughlin and J. Templeton, "Hip fractures in patients with Parkinson's disease," Clin. Orthop. Relat. Res., vol. 148, pp. 192-195, (1980)

15. A. Hosseinzadeh, M. Khalili, B. Sedighi, S. Iranpour, and A. A. Haghdoost, "Parkinson's disease and risk of hip fracture: systematic review and meta-analysis," Acta Neurol. Belg., vol. 118, no. 2, pp. 201-210, (2018)

16. N. Giladi, H. Shabtai, E. S. Simon, S. Biran, J. Tal, and A. D. Korczyn, "Construction of freezing of gait questionnaire for patients with Parkinsonism," Park. Relat. Disord., vol. 6, no. 3, pp. 165-170, (2000)

17. N. Giladi et al., "Validation of the Freezing of Gait Questionnaire in patients with Parkinson's disease," Mov. Disord., vol. 24, no. 5, pp. 655-661, (2009)

18. S. Vercruysse, M. Gilat, J. M. Shine, E. Heremans, S. Lewis, and A. Nieuwboer, "Freezing beyond gait in Parkinson's disease: A review of current neurobehavioral 
evidence," Neurosci. Biobehav. Rev., vol. 43, pp. 213-227, (2014)

19. E. Matar, J. M. Shine, S. L. Naismith, and S. J. G. Lewis, "Using virtual reality to explore the role of conflict resolution and environmental salience in Freezing of Gait in Parkinson's disease," Park. Relat. Disord., vol. 19, no. 11, pp. 937-942, (2013)

20. J. Nantel, J. C. McDonald, S. Tan, and H. Bronte-Stewart, "Deficits in visuospatial processing contribute to quantitative measures of freezing of gait in Parkinson's disease," Neuroscience, vol. 221, no. 2012, pp. 151-156, (2012)

21. J. M. Shine et al., "Freezing of gait in Parkinson's disease is associated with functional decoupling between the cognitive control network and the basal ganglia," Brain, vol. 136, no. 12, pp. 3671-3681, (2013)

22. S. Vercruysse et al., "Abnormalities and cue dependence of rhythmical upper-limb movements in Parkinson patients with freezing of gait," Neurorehabil. Neural Repair, vol. 26, no. 6, pp. 636-645, (2012)

23. Q. J. Almeida, L. R. Wishart, and T. D. Lee, "Disruptive influences of a cued voluntary shift on coordinated movement in Parkinson's disease," Neuropsychologia, vol. 41, no. 4, pp. 442-452, (2003)

24. A. Nieuwboer, S. Vercruysse, P. Feys, O. Levin, J. Spildooren, and S. Swinnen, "Upper limb movement interruptions are correlated to freezing of gait in Parkinson's disease," Eur. J. Neurosci., vol. 29, no. 7, pp. 1422-1430, (2009)

25. S. Vercruysse et al., "The neural correlates of upper limb motor blocks in Parkinson's disease and their relation to freezing of gait," Cereb. Cortex, vol. 24, no. 12, pp. 3154 3166, (2014)

26. A. J. Williams, D. S. Peterson, M. Ionno, K. A. Pickett, and G. M. Earhart, "Upper extremity freezing and Dyscoordination in Parkinson's disease: Effects of amplitude and cadence manipulations," Parkinsons. Dis., vol. 2013, (2013)

27. E. E. Tripoliti et al., "Automatic detection of freezing of gait events in patients with Parkinson's disease," Comput. Methods Programs Biomed., vol. 110, no. 1, pp. 12-26, (2013)

28. B. T. Cole, S. H. Roy, and S. H. Nawab, "Detecting freezing-of-gait during unscripted and unconstrained activity," Proc. Annu. Int. Conf. IEEE Eng. Med. Biol. Soc. EMBS, pp. 5649-5652, (2011)

29. S. Rezvanian and T. E. Lockhart, "Towards real-time detection of freezing of gait using wavelet transform on wireless accelerometer data," Sensors (Switzerland), vol. 16, no. 4, (2016)

30. J. F. Hafer, S. G. Provenzano, K. L. Kern, C. E. Agresta, J. A. Grant, and R. F. Zernicke, "Measuring markers of aging and knee osteoarthritis gait using inertial measurement units," J. Biomech., vol. 99, p. 109567, (2020)

31. H. Cantú, J. N. Côté, and J. Nantel, "A new method based on quiet stance baseline is more effective in identifying freezing in Parkinson's disease," PLoS One, vol. 13, no. 11, pp. 1-14, (2018)

32. J. Nantel, C. de Solages, and H. Bronte-Stewart, "Repetitive stepping in place identifies and measures freezing episodes in subjects with Parkinson's disease," Gait Posture, vol. 34, no. 3, pp. 329-333, (2011)

33. J. D. Schaafsma, Y. Balash, T. Gurevich, A. L. Bartels, J. M. Hausdorff, and N. Giladi, "Characterization of freezing of gait subtypes and the response of each to levodopa in Parkinson's disease," Eur. J. Neurol., vol. 10, no. 4, pp. 391-398, (2003) 